\title{
Effective Treatment of Undiferentiated Pleomorphic Sarcoma Using Gemcitabine: A Case Report
}

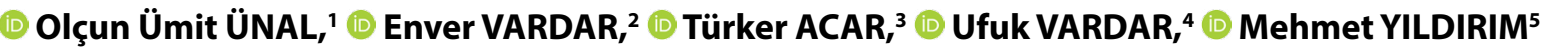

\author{
'Department of Medical Oncology, SBU Bozyaka İzmir Training and Research Hospital, İzmir-Turkey \\ ${ }^{2}$ Department of Pathology, SBU Bozyaka İzmir Training and Research Hospital, İzmir-Turkey \\ ${ }^{3}$ Department of Radiology, SBU Bozyaka İzmir Training and Research Hospital, İzmir-Turkey \\ ${ }^{4}$ Department of Medical Education, Ege University Faculty of Medicine, Izmir-Turkey \\ ${ }^{5}$ Department of General Surgery, SBU Bozyaka Training and Research Hospital, İzmir-Turkey
}

\begin{abstract}
SUMMARY
Undifferentiated pleomorphic sarcoma (UPS) is most frequently observed in the extremities but rarely in the retroperitoneum. The present treatment for patients with metastatic or unresectable UPS in the first-line setting includes doxorubicin administration with or without ifosfamide. We present a case of a 68-year-old woman who presented with a retroperitoneal giant mass. The patient underwent surgery, the mass was removed, and the final diagnosis was UPS. The postoperative stage included soft tissue metastases. After three cycles of gemcitabine chemotherapy, computed tomography findings revealed the absence of soft tissue metastases. The patient has remained on regular follow-up over the past two years since chemotherapy with no symptoms of disease recurrence. The findings of this case demonstrate that patients with retroperitoneal UPS may benefit from single-agent gemcitabine chemotherapy.

Keywords: Cardiac failure; complete remission; gemcitabine; undifferentiated pleomorphic sarcoma.

Copyright $\odot$ 2018, Turkish Society for Radiation Oncology
\end{abstract}

\section{Introduction}

Among retroperitoneal sarcomas, which represent $10 \%-15 \%$ of all soft tissue sarcomas (STSs), liposarcoma is the most frequently occurring histotype.[1] Undifferentiated pleomorphic sarcoma (UPS) is the second-most common subtype of retroperitoneal sarcoma, and literature is limited regarding this disease.

Presently, the management of patients with metastatic STS usually includes palliative chemotherapy. Doxorubicin and ifosfamide are the most active agents against sarcomas, with a response rate of approximately $25 \%$. [2] However, cardiac failure remains a contraindication of anthracycline use. Other agents with activity against soft tissue sarcoma contain dacarbazine, gemcitabine, docetaxel, vinorelbine, and methotrexate.[2]
Gemcitabine is an analog of deoxycytidine that has demonstrated clinical activity in a variety of solid tumors, including bladder, lung, and pancreatic carcinomas. [3] In addition, gemcitabine has shown activity against sarcoma cell lines xenografts in a preclinical study.[4] A previous publication has also suggested moderate gemcitabine activity in patients with soft tissue sarcomas.[5]

Herein we report a radiological complete response following gemcitabine chemotherapy in an elderly woman with metastatic UPS that arose in the retroperitoneal area.

\section{Case Report}

A 68-year-old woman presented with gradually progressive history of abdominal fullness at our hospital in 


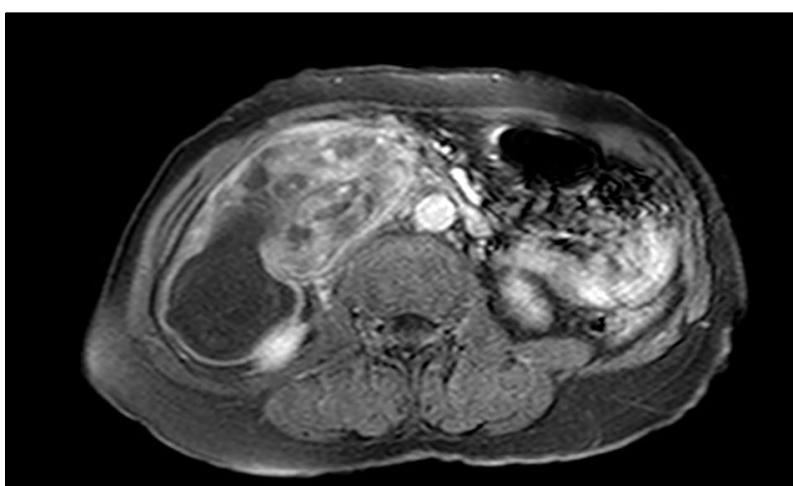

Fig. 1. Magnetic resonance imaging (MRI) scan of the abdomen before the initiation of surgery shows a heterogeneous mass.

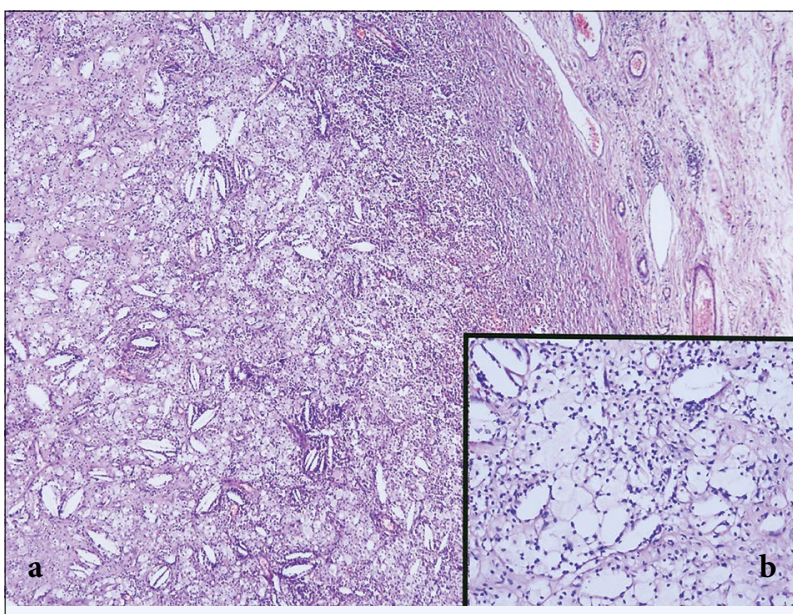

Fig. 2. This retroperitoneal mass was comprised predominantly of low-grade sarcoma with characterized minimally irregular peripheral border and predominant histiocyte-like foamy cells (a). A majority of the tumor had the same histologic appearance of well-differentiated areas and cholesterol cyrstals may occasionally be seen in cytoplasm of foamy tumor cells. (b-inset) (HE stain).

July 2015. On abdominal physical examination, a giant mass was palpated in her right abdomen. Abdominal magnetic resonance imaging (MRI) revealed a tumor measuring approximately $14 \times 12 \times 7 \mathrm{~cm}$ in size in the retroperitoneum (Fig. 1). In August 2015, the patient underwent exploratory laparotomy for removal of the tumor. En bloc resection was successfully performed without any remaining tumor. A diagnosis of UPS (Grade 2 according to the French Fédération Nationale des Centres de Lutte Contre le Cancer grading system) was confirmed by a pathologist. Immunohistochemical analysis was performed by incubation with the following primary antibodies: pan-cytokeratin, smooth

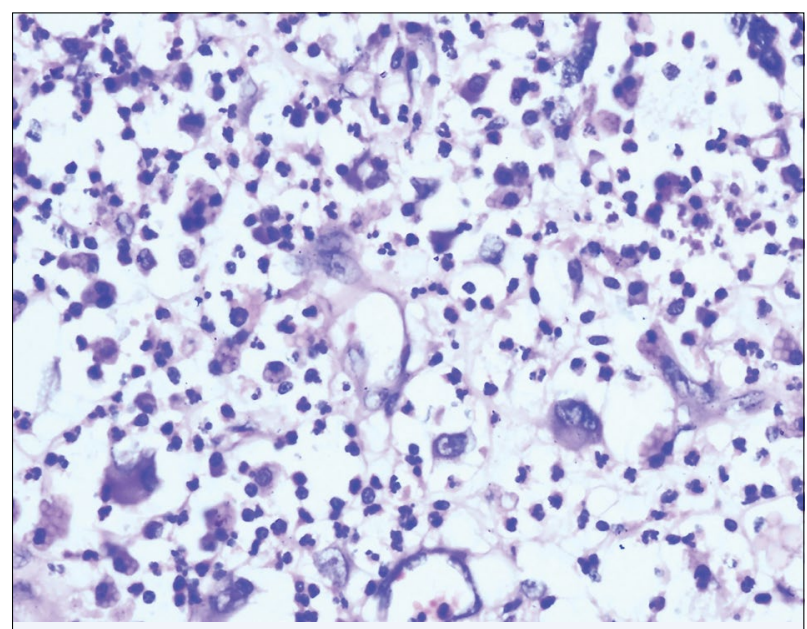

Fig. 3. Focally, these cytologically pleomorphic tumor cells were present and were associated with inflammatory cells. Focal nuclear atypia and pleomorphism in the absence of mitotic activity or necrosis is of uncertain prognostic significance. (HE stain-x400).

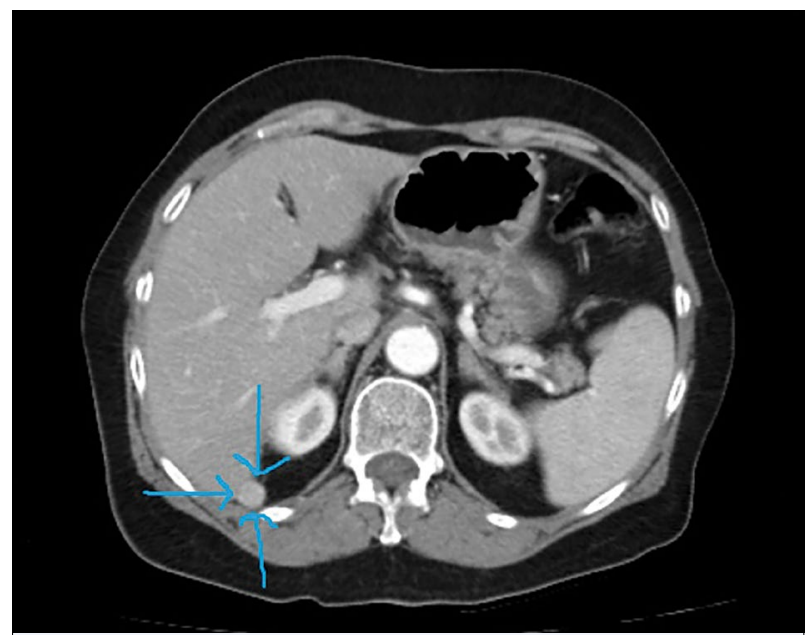

Fig. 4. Postoperative computed tomography (CT) scan of abdomen shows a metastatic mass.

muscle actin, desmin, vimentin, melan-A, S100, CD68, and HMB-45. Immunohistochemical analysis revealed positivity for vimentin and there no staining was observed for pancytokeratin, desmin, HMB-45, MelanA, S-100, and CD117 (Figs. 2 and 3). In the medical oncology polyclinic, a physical examination that had been performed upon admission revealed cardiac auscultation with irregular heart sounds and a Levine III/IV systolic murmur at the point of maximum intensity, which was located at the apex. Edema was present in the lower extremities; in addition, the patient's Eastern Cooperative Oncology Group (ECOG) performance 


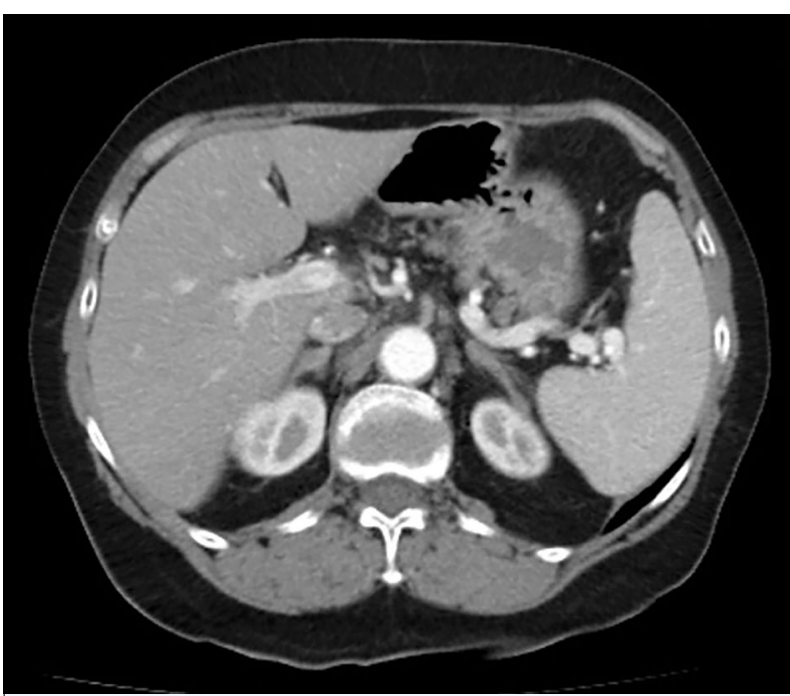

Fig. 5. Computed tomography (CT) scan of abdomen following gemcitabine chemotherapy.

status was 2. Echocardiography revealed reduced left ventricular systolic function, with a left ventricular ejection fraction of $40 \%$. Soft tissue metastasis was observed in the postoperative computed tomography (CT) scan (Fig. 4). The patient received palliative chemotherapy with three cycles of gemcitabine (1000 $\mathrm{mg} / \mathrm{m} 2 /$ half hour [days 1,8 , and 15 ] every 28 days), from September 2015 to December 2015. The therapy was well-tolerated, and the patient experienced only fatigue. An abdominopelvic CT scan revealed a complete response (Fig. 5) according to the Response Evaluation Criteria in Solid Tumors criteria. Gemcitabine chemotherapy was completed after six cycles, and the patient did not receive further treatment. The patient remained alive without tumor recurrence at the followup appointment in April 2018.

\section{Discussion}

We describe the case of a patient with metastatic UPS who experienced a complete radiological response. She maintained long-term disease control after the completion of gemcitabine chemotherapy.

UPS is a rare soft tissue neoplasm that was first reported in 1977 and was further characterized by Weiss in 1978.[6] This disease is extremely rare in the retroperitoneal region. However, UPS, which primarily occurs in the retroperitoneum, has a poorer prognosis than do sarcomas that occur at other sites.

Knowledge regarding retroperitoneal STS's clinical course and management is limited because of the low prevalence. The clinical course of retroperitoneal STS is determined by tumor size, grade, and evidence of metastasis. The primary treatment for retroperitoneal STS is surgical resection. Adjuvant radiation therapy may be effective in controlling loco-regional recurrence; however, with respect to adjuvant and palliative systemic chemotherapy, data exist only from soft tissue sarcomas at other sites of the body. Anthracyclinebased chemotherapy may be effective, but its effect on overall survival is yet to be reported. In the present case, two months following surgery, soft tissue metastasis developed and very good response was obtained from a single-agent gemcitabine regimen.

Gemcitabine is known to have a high level of activity in leiomyosarcoma and modest activity in other soft tissue sarcomas.[2] A single report of a patient with a soft tissue angiosarcoma who achieved complete remission has been published in the literature [7]; however, whether complete remission can be achieved for UPS and other STS is yet to be reported.[8,9]

To our knowledge, we report the first documented case of metastatic UPS that originated in the retroperitoneal area, wherein the patient achieved a complete radiographic response to a single-agent gemcitabine regimen. This regimen has potential advantages over frequently used anthracine-based regimen since it is associated with less cardiotoxicity, improved tolerability, and the convenience of outpatient administration. These features may prove especially useful in patients who are elderly, have comorbidities, or are afflicted with retroperitoneal sarcomas.

\section{Conclusion}

In conclusion, this case suggests that gemcitabine chemotherapy may be an alternative therapy for patients with retroperitoneal soft tissue sarcoma.

Peer-review: Externally peer-reviewed.

Conflict of Interest: The authors declare no potential conflicts of interest.

Authorship contributions: Concept - O.Ü.Ü.; Design O.Ü.Ü.; Supervision - M.Y.; Data collection \&/or processing - E.V.; Analysis and/or interpretation - T.A.; Literature search - U.V.; Writing - O.Ü.Ü., U.V.; Critical review O.Ü.Ü., E.V.

\section{References}

1. Trans-Atlantic Retroperitoneal Sarcoma Working Group (TARPSWG). Management of metastatic retroperitoneal sarcoma: a consensus approach from the Trans-Atlantic Retroperitoneal Sarcoma Working 
Group (TARPSWG). Ann Oncol 2018;29(4):857-71.

2. Besiroglu M, Dane F, Ciltas A, Benekli M. Systemic chemotherapy of advanced soft tissue sarcomas. J Onc Sci 2017;3(2):66-70.

3. Braakhuis BJ, Ruiz van Haperen VW, Boven E, Veerman G, Peters GJ. Schedule-dependent antitumor effect of gemcitabine in in vivo model system. Semin Oncol 1995;22(4 Suppl 11):42-6.

4. Merimsky O, Meller I, Flusser G, Kollender Y, Issakov $\mathrm{J}$, Weil-Ben-Arush M, et al. Gemcitabine in soft tissue or bone sarcoma resistant to standard chemotherapy: a phase II study. Cancer Chemother Pharmacol 2000;45(2):177-81.

5. Svancárová L, Blay JY, Judson IR, van Hoesel QG, van Oosterom AT, le Cesne A, et al. Gemcitabine in advanced adult soft-tissue sarcomas. A phase II study of the EORTC Soft Tissue and Bone Sarcoma Group. Eur J Cancer 2002;38(4):556-9.
6. Weiss SW, Enzinger FM. Malignant fibrous histiocytoma: an analysis of 200 cases. Cancer 1978;41(6):225066.

7. Kajihara I, Maeda S, Yamada S, Izumi K, Masuguchi S, Fukushima $S$, et al. Biweekly gemcitabine therapy induces complete remission in cutaneous angiosarcoma resistant to multiple anticancer drugs. J Dermatol 2015;42(12):1197-8.

8. Von Burton G, Rankin C, Zalupski MM, Mills GM, Borden EC, Karen A. Phase II trial of gemcitabine as first line chemotherapy in patients with metastatic or unresectable soft tissue sarcoma. Am J Clin Oncol 2006;29(1):59-61.

9. Ferraresi V, Ciccarese M, Cercato MC, Nuzzo C, Zeuli M, Di Filippo F, et al. Gemcitabine at fixed dose-rate in patients with advanced soft-tissue sarcomas: a monoinstitutional phase II study. Cancer Chemother Pharmacol 2008;63(1):149-55. 